\title{
Rechtskulturen und Rechtssysteme im weltweiten Vergleich Eine internationale Tagung des Instituto de Investigaciones Juridicas, Mexico City (Februar 2004)
}

\author{
Von Markus Kotzur, Bayreuth / Leipzig
}

\section{I. $\quad$ Einleitung}

Die Transformation der latein- bzw. iberoamerikanischen Staaten und Gesellschaften hin zu Demokratie, Rechts- und Verfassungsstaatlichkeit ist ein erfolgversprechender, aber längst noch nicht abgeschlossener Prozess. Er bedarf von rechtlicher wie politischer Seite der Unterstützung vor allem durch Europa und die Vereinigten Staaten. Gewiss hilfreich sind die seit dem ersten Zusammentreffen in Rio de Janeiro (1999) institutionalisierten EULateinamerika-Gipfel. Die jüngste Gipfelkonferenz (2004) fand in Mexico City statt, doch schon im Vorfeld war deutlich, dass manche hochgesteckten Erwartungen enttäuscht würden. ${ }^{1}$ Seien es erste Fortschritte bei der Bekämpfung des Drogenhandels auf der einen oder politisch-soziale Krisenszenarien - wie derzeit in Mexiko unter Präsident V. Fox und seiner ambitionierten, aber umstrittenen Gattin M. Sahagun - auf der anderen Seite: USA und Europäische Union beschäftigen sich seit dem 11. September 2001 vornehmlich mit Afghanistan, dem Irak und dem Nahen Osten; sie verlieren dabei Latein- bzw. Iberoamerika mitunter allzu rasch aus den Augen. Gibt es auf Seiten der Jurisprudenz hier ein Parallelphänomen? Die europäische Rechtswissenschaft hat die Demokratisierungs- und Reformbemühungen auf dem amerikanischen Kontinent zwar mit kritischem Interesse begleitet und wollte häufig als Vorbild wirken. Manche eurozentrische Selbstherrlichkeit blieb freilich nicht aus. ${ }^{2}$ Umso dringender sind gemeinsame Gesprächsforen, die dem transatlantischen Rechtsgespräch stetig neue Impulse geben und allzu einseitige perspektivische Verengungen zu korrigieren helfen. D. Valdés und das von ihm geleitete Instituto de Investigaciones Jurídicas an der Universidad Nacional Autónoma de México haben diesbezüglich schon mehrfach Pionierarbeit geleistet. ${ }^{3}$ Morlok (Hrsg.), Die Welt des Verfassungsstaates, 2001, S. $189 \mathrm{ff}$. wirklichkeit - Erste Ergebnisse einer Bürgerbefragung des Instituto de Investigaciones Juridicas, Mexico City, in: VRÜ 37 (2004), S. 88 ff. 
Die „Natur- und Kulturbedingungen“4 ${ }^{4}$ des Verfassungsstaates in weltweitem Verglich zu erschließen, gehört zu den Hauptanliegen des Instituts. Methodisch stützt es sich dabei auf den insbesondere von $P$. Häberle entwickelten kulturwissenschaftlichen Ansatz ${ }^{5}$. In allen Disziplinen des Rechts sollen gemeinrechtliche Strukturen nachgewiesen, Gemeinsamkeiten regionaler Verantwortungsgemeinschaften konturiert, universelle Elemente einer globalen Rechtskultur entwickelt werden. Ein Stichwort gibt etwa das gemeinamerikanische als Pendant zum gemeineuropäischen Recht. ${ }^{6}$ Im Februar 2004 konnte das Instituto de Investigaciones Jurídicas erstmals in der latein-/iberoamerikanischen Staatenwelt einen umfassend angelegten Kongress zum ,weltweiten Vergleich der Rechtskulturen und Rechtssysteme" ausrichten, der alle rechtswissenschaftlichen Teildisziplinen eingebunden und an die eintausend Rechtswissenschaftler aus aller Welt für eine Woche intensiver Arbeit in Mexiko City zusammengeführt hat. Kongressprogramm und Tagungsergebnisse seien im folgenden vorgestellt.

\section{Die zentralen Tagungsthemen}

Der einwöchige Kongress verfolgte eine ehrgeizige Zielsetzung. Im internationalen Vergleich sollten nicht nur die unterschiedlichen Rechtskulturen einbezogen, sondern auch intradisziplinär die einzelnen Teilfelder rechtswissenschaftlichen Forschens ausgeleuchtet werden. Zu diesem Zwecke wurden insgesamt zwölf Arbeitsgruppen gebildet, die sich teils am klassischen Profil der juristischen Disziplinen, teils an hochaktuellen Fragestellungen orientierten. Pro Arbeitsgruppe respektive ,discussion panel“ präsentierten an fünf aufeinander folgenden Tagen etwa 30 Wissenschaftler in Vortrag und Diskussion ihre Arbeitsergebnisse. Bei mehr als 300 Referenten und weit über 500 sonstigen Kongressteilnehmern aus Studentenschaft, Wissenschaft und Praxis wird klar: Der Veranstaltung ging es in erster Linie um eine - zu recht - selbstbewusste Standortbestimmung und Außendarstellung dessen, was die latein-/iberoamerikanischen Wissenschaftlergemeinschaften angesichts tiefgreifender Transformations- und Reformprozesse der beiden letzten Jahrzehnte leisten konnten. So war denn auch die lingua franca im Wissenschaftsgespräch einmal nicht

H. Heller, Staatslehre, 6. Aufl. 1983, S. 189.

P. Häberle, Verfassungslehre als Kulturwissenschaft, 1982, 2. Aufl. 1998, S. 2 ff.; siehe dort den Verweis auf den offenen Kulturbegriff von C. Kluckhohn, The Concept of Culture, in: E. A. Schuler et al. (eds.), Readings in sociology, $4^{\text {th }}$ ed. 1971, S. 74 ff.; aus der Folgeliteratur heute in globaler Perspektive Ph. Mastronardi, Recht und Kultur: Kulturelle Bedingtheit und universaler Anspruch des juristischen Denkens, ZaöRV 61 (2001), S. 61 ff.

6

$P$. Häberle, Mexiko - Konturen eines Gemeinamerikanischen Verfassungsrechts - ein ius commune Americanum, JöR 52 (2004), S. 581 ff. 
vorwiegend das Englische, sondern das Spanische. ${ }^{7}$ Dem Ideal einer lebendigen „,debating society" können in zeitlicher wie personeller Hinsicht so groß angelegte Kongresse indes nur bedingt gerecht werden. Sie geben eher erste Diskussionsanstöße, die anhand der schriftlichen Vortragspublikationen ausgewertet und in späteren - stärker auf wechselseitiges Lernen hin ausgerichteten - kleineren Folgetagungen weiterentwickelt, auch kulturspezifisch ausdifferenziert werden können. Besonders neugierig macht diesbezüglich eine von D. Valdés und dem Instituto de Investigaciones Jurídicas für das Jahr 2005 geplante Osteuropatagung. Doch zurück zu den zentralen Arbeitsthemen des hier vorzustellenden Kongresses.

Das erste „Panel“ galt dem Verwaltungsrecht (Derecho Administrativo), das zweite dem Verfassungsrecht (Derecho Constitucional), das dritte dem internationalen Recht und spezifisch der internationalen Sicherheit einschließlich des Kampfes gegen Drogenhandel und weltweit agierende Terrorgruppen (Derecho y Seguridad Internacional). Die Arbeitsgruppen vier, fünf und sechs hatten mit dem Strafrecht (Derecho Penal) und dem Zivilrecht (Derecho Privado) zwei klassische, mit dem Sozialrecht (Derecho Social) eine jüngere, für die wirtschaftliche Prosperität und politische Stabilität der latein-/iberoamerikanischen Staatenwelt besonders bedeutsame Teildisziplin(en) der Jurisprudenz zum Gegenstand. Zwei aktuelle Themen wurden von der Arbeitsgruppe neun und der Arbeitsgruppe zwölf behandelt: zum einen künstliche Intelligenz und Recht (Inteligencia Artificial y Derecho) gedacht war auch an das Internet und die vielfältigen, mit seiner Nutzung verbundenen Probleme (zivil- und international-privatrechtliche Aspekte des Internethandels, Schutz des geistigen Eigentums im weltweiten Netz, Jugendschutz, wirksame Mittel gegen die Weiterverbreitung strafrechtlich relevanter Daten und Bilder); zum anderen Gesundheit und Recht (Salud y Derecho), von Herausforderungen der Bioethik bis hin zu effektivem Krankenversicherungsschutz. Vier Arbeitsgruppen galten schließlich den übergreifenden Grundlagenthemen: Arbeitsgruppe sieben der Rechtsphilosophie und Rechtstheorie (Filosofía y Teoriá del Derecho), Arbeitsgruppe acht der Rechtsgeschichte (Historia del Decho), Arbeitsgruppe zehn der Methodologie des Rechtsvergleichs (Metodología del Derecho Comparado) und Arbeitsgruppe elf der Rechtssoziologie (Sociología del Derecho).

Die einzelnen Vorträge und Podiumsdiskussionsbeiträge können hier nur in thematisch strukturierter, knapper Auswahl vorgestellt werden. Interessant sind dabei vor allem jene universellen Problemstellungen, die in nahezu allen Rechtskulturen einen hoch aktuellen Gegenstand wissenschaftlicher Auseinandersetzung bilden. Begonnen sei mit dem Verfasinst/evacad/eventos/2004/0902/ publiziert und auch auf CD-ROM erhältlich. Eine Publikation in wohl mehreren Tagungsbänden ist ebenfalls geplant. 
sungsrecht, das seinerseits unter dem methodischen Leitmotiv des Verfassungsvergleichs stand. P. Häberle ${ }^{8}$ zeichnete in seinem von H. Fix-Zamudio und P. de Vega kommentierten Einleitungsreferat das Bild national-verfassungsstaatlich respektive regional radizierter, international ausgreifender Verantwortungsgemeinschaften. Ihr einigendes Band seien einige wenige, rechtsvergleichend $\mathrm{zu}$ erschließende und bei aller kulturellen Partikularität universalisierbare Grundprinzipien. Damit waren drei Themenkomplexe erschlossen, die für die weitere Diskussion der verfassungsrechtlichen und der rechtsvergleichenden Arbeitsgruppe eine zentrale Rolle spielen sollten: der Schutz der Menschenwürde und der Menschenrechte in staatlich wie staatenübergreifend verfassten politischen Gemeinschaften ${ }^{9}$; der Rechtsstaat bzw. sein weniger staatszentriertes angloamerikanisches Pendant, die Rule of Law, als universelles Strukturprinzip des Typus Verfassungsstaat und Richtschnur für die internationale Staatengemeinschaft ${ }^{10}$; schließlich die Verfassungsrechtssprechung und ihr nahezu weltweiter Siegeszug. ${ }^{11}$ Auf verwaltungsrechtlichem Sektor sind Internationalisierung und Globalisierung die Rahmenbedingungen, die eine Neuorientierung vieler Spezialbereiche des besonderen Verwaltungsrechts notwendig machen (etwa das Energieoder Telekommunikationsrecht) ${ }^{12}$, die zugleich aber auch rechtsethische Reflexion erfordern $^{13}$. Wie ein roter Faden durchzieht seit dem 11. September eine Neuausrichtung der öffentlichen Sicherheit die Diskussion. Hier treffen sich nationalstaatliches Verfassungs/Verwaltungsrecht ${ }^{14}$ und internationales Recht. ${ }^{15}$ Der weltweite Kampf gegen den Terroris-

P. Häberle (Deutschland), Derecho constitucional nacional, „uniones de estados“ régionales y el derecho internacional como derecho universal de la humanidad: convergencias y divergencias.

A. Buenrostro Cevallos (Mexiko), Seguridad pública y derechos humanos: un ejercicio ciudadano (zum Nachweis für diese und die folgenden Beiträge siehe oben Fn. 7); M. Beltrán Gaos (Spanien), La protección descentralidada de los derechos humanos en México y en España; $R$. Daniel Pizarro, La protección de la dignidad humana frente a la agresión de los medios masivos de comunicación social por informaciones inexactas o agraviantes; aus europäischer Sicht siehe schließlich H.-P. Schneiders Beitrag zur Universalität/Universalisierbarkeit der Menschenrechte.

J. L. Prado Maillard (Mexiko), Nuevos paradigmas de la separación de poderes ; J. Alfonso da Silva (Brasilien), Direito Constitucional comparado e processo de reforma do estado.

H. Botha (Südafrika), Comparative Law and constitutional adjudication; R. Gustavo Ferreyra (Argentinien), Corte Suprema de Justicia Argentina y control de constitucionalidad; F. JulienLaferriere (Frankreich), Las insuficiencias del control de la constitucionalidad de las leyes en Francia.

F. Buzzo (Argentinien), El regimen legal de la energia electrica en la Republica Argentina; $J$. Fernández Ruiz (Mexiko), La reforma energética; C. Chinchilla Marin (Spanien), El régimen jurídico de las telecomunicaciones: Del servicio público y la gestión en monopolio al servicio de interés general prestado en régimen de competencia.

13 J. González Perez (Spanien), Etica pública: La corrupción, la ética y el derecho; J. R. Olivos Campos (Mexiko), Lo público de la ética pública. 
mus, in den Fällen Afghanistan und Irak, auch im Nahostkonflikt höchst aktuell, beherrscht die völkerrechtliche Diskussion. In diesen Kontext gehören aber auch die Drogenprävention und das Ringen um Legitimationskriterien für die humanitäre Intervention. ${ }^{16}$ Deutlich wird, dass zu Beginn des 21. Jahrhunderts die innere und äußere, aber auch die soziale Sicherheit die großen, interdependenten Reformthemen des Verfassungsstaates formulieren. ${ }^{17}$ Freiheits- und Sicherheitsvorsorge sind zentrale Legitimationsvoraussetzungen und grundrechtlich abgesicherter Doppelzweck verfassungsstaatlicher Herrschaftsorganisation. Die Diskussion in den verwaltungs-, verfassungs- und völkerrechtlichen Arbeitsgruppen hat eindrucksvoll gezeigt, wie fragil die Balance von Freiheit und Sicherheit unter dem Eindruck der aktuellen Entwicklungen geworden ist. Dass die Freiheit aber nicht einem umfassenden Präventionsstaat geopfert werden darf, war ihr unzweideutiges Postulat. ${ }^{18}$

Gesellschaftlicher Wandel widerspiegelte sich in den Debatten des Zivilrechts (etwa zu den gleichgeschlechtlichen Lebenspartnerschaften - gerade ein gesellschaftspolitisch so kontrovers diskutiertes (Reiz-)Thema ist auf umfassenden Kulturvergleich einschließlich der religiösen Dimension angewiesen! ${ }^{19}$ ). Die Globalisierung ist eines der Leitthemen, denen es sich in seinen Methoden und Inhalten stellen muss. Je selbstverständlicher internationale Wirtschaftskooperation das Tagesgeschäft von Unternehmen und Großkonzernen bestimmt, um so drängender wird, was im Recht der Europäischen Gemeinschaften ausdrücklich festgeschrieben ist: Harmonisierung und Rechtsangleichung. Das klassische internationale Privatrecht als Ausgleichsordnung konfligierender Rechtssysteme (Conflict of Laws) vermag eine nur unzureichende Antworten auf die aktuellen Herausforderungen zu geben. Von daher ist es nicht verwunderlich, dass in Latein-/Iberoamerika Bemühungen zur Vereinheitlichung des Zivilrechts durch die Europäische Union auf großes Interesse

A. Camen (Schweiz), El derecho internacional humanitario y la seguridad internacional; $G$. de Greiff (Brasilien), Terrorismo y seguridad nacional. El derecho internacional que hereda el siglo XXI; A. González Carrillo (Mexiko), Soberanía y regulación contra el terrorismo: Pasado y presente de la cooperación internacional de México ante las amenazas a la paz; M. Kotzur (Deutschland), The Struggle against Terrorism - Chances and Limits of modern public international law.

L. Armendáriz (Spanien), La intervención por razones de humanidad.

Etwa G. Katrougalos (Griechenland), The European Model of Protection of Social Rights; $G$. Mendizábal Bermúdez (Mexiko), Estudio comparativo de los sistemas de seguridad social con tradidión a y de tipo Berveridgiano, al ejemplo de dos países : Austria - Méxiko.

Aus völkerrechtlicher Perspektive $P$. C. Revilla Montoya (Peru), Consequencias Jurídicas del ataque a Irak: Reformulación del sistema de seguridad internacional.

P. J. Viladrich (Spanien), Las secuencas de la sexualidad humana y sus asociaciones en la expresión canónica del matrimonio. En busca de un lugar de encuento entre las grandes culturas y tradicuiones sobre el matrimonio. 
stoßen. ${ }^{20}$ Vor allem aus mexikanischer Perspektive mag das Binnenmarktkonzept der EG inspirierende Vorbildfunktion für die weitere Ausgestaltung der Wirtschaftskooperation im Rahmen der NAFTA haben und das Denken in grenzüberschreitenden Rechtsräumen nachhaltig befruchten.

Grenzüberschreitungen in vielfacher Hinsicht werden auch für das Strafrecht notwendig, sie umgreifen das materielle Strafrecht wie das Strafprozessrecht. Der Internationale Strafgerichtshof gibt dafür ein signifikantes Beispiel. Das Völkerstrafrecht bedarf heute konsequenter Implementierung in die nationalen Rechtsordnungen. Umgekehrt tut die Entwicklung universeller strafprozessualer Regelungen Not, um ein Mindestmaß an Kohärenz zwischen national-verfassungsstaatlicher und internationaler Strafrechtspflege herzustellen. $^{21}$ Der Überblick über die öffentlich-rechtlichen, zivil- und strafrechtlichen Arbeitsgruppen bestätigt: Bei aller angedeuteten Themenvielfalt, bei manchen methodischen Divergenzen wird ein dringendes Bedürfnis nach komparatistischer Analyse deutlich. Sie ist das disziplinenübergreifende, einigende Band, früh und in großer Tradition durch das Zivilrecht kontinentaleuropäischer Prägung ebenso wie durch das common law ausgeformt, heute durch das Straf-, Verfassungs- und Verwaltungsrecht konsequent aufgegriffen. ${ }^{22}$ Nur der Rechtsvergleich kann im weltweiten Aufeinanderangewiesensein der Rechtssysteme und Rechtskulturen der Jurisprudenz ein tragfähiges methodisches Handwerkszeug liefern, so schon D. Valadés bei seinen Einführungsworten. ${ }^{23}$ Vergleichend angelegt sein müssen auch die Grundlagendisziplinen selbst. Der Rechtsphilosophie, der Rechtstheorie, der Rechtsgeschichte und auch der Rechtssoziologie ist ein vergleichendes Moment immanent. ${ }^{24}$ Sie müssen sowohl interdisziplinär als auch komparatistisch ausgreifen. Sie sind vor allem aber die Voraussetzung dafür, dass Rechtsvergleich als umfassender, bei aller Selektivität dennoch rationalisierbarer Kulturvergleich gelingen kann. Eines haben die Diskussionen immer wieder deutlich gemacht: Allein bei den Rechtstexten, Gerichtsentscheidungen oder Theorieentwürfen der nationalen Wissenschaftlergemeinschaften stehen zu blei-

J. de Jesus Borjón Nieto (Mexiko), Jursidicción universal y jurisdicción interna : ¿Cómo salvar escollos?

L. Pegoraro (Italien), Derecho Constitucional y método comparativo ; A. Ma. Hernández (Argentinien), La influencia de la constitucion Norteamericana en el federalismo Argentino y de la constucción Española de 1978 en el constitucionalismo provincial; D. de Douza de Brito (Portugal), Motivações e tendêcias póstmodernas do direito comparado e as filosofias ds globalização.

Siehe darüber hinaus auch sein Vorwort zu Bd. 1 der Zeitschrift „Cuestiones Constitucionales“ 1999, hrsgg. vom Instituto de Investigaciones Juridicas der Universidad Nacional Autónoma de Méxiko (besprochen von H.-R. Horn, in: VRÜ 33 (2000), S. 264 ff., sowie sein Tagungsreferat zum Thema „La substitución presidencial en México y en derecho comparado”.

Vgl. etwa E. Grün (Argentinien), La globalizacíon del derecho: un fenómeno sistémico y cibernético. 
ben, genügt nicht. Sie sind Ausgangspunkt des Rechtsvergleichs, der aber kontextsensibel ${ }^{25}$ angelegt sein muss, indem er die Makrodimensionen der politischen, sozialen, ökonomischen, ökologischen, historischen - kurz der kulturellen Rahmenbedingungen - mit einbezieht.

\section{Eine erste Bewertung der Tagungsergebnisse}

Ein so umfassend angelegter Kongress wie der hier vorgestellte muss sich seiner Chancen und Grenzen bewusst sein. Zunächst zu den Grenzen: Bei einer Zusammenschau der wichtigsten Rechtskulturen und nahezu aller Rechtsdisziplinen bleibt vieles nur angedeutet, manches noch allzu abstrakt und allgemein, bisweilen schemenhafte Globalvision. Beim komparatistischen Zugriff besteht vor allem die bereits angesprochene Gefahr unreflektierter Selektion. Damit die Auswahl der Vergleichstopoi nicht zu willkürlichem Eklektizismus wird, müssen das Vorverständnis und die Auswahlkriterien offengelegt werden. Das Diskussionsforum in Mexico City wollte und konnte helfen, die kulturbedingt unterschiedlichen Vorverständnisse transparent zu machen. Ein „Dialog der Rechtskulturen und Rechtsysteme“ ist zunächst einmal ein Dialog über divergierende Vorverständnisse. Damit beginnt der - von der Rechtswissenschaft methodisch zu bändigende - Lernprozess. Und damit wären auch die Chancen, der letztlich doch reiche Kongressertrag angesprochen. Im Folgenden eine thesenartige Zusammenfassung.

1. Rechtsvergleichendes Arbeiten setzt einen beständigen Kommunikationsprozess voraus. Dieser bedarf öffentlichkeitswirksamer Foren. ${ }^{26}$ Insoweit kann und will die hier vorgestellte Tagung nur der erste Anstoß für einen „Dialog der Rechtskulturen“ sein, den es weiterhin fortzusetzen und mit Blick auf neue Dialogpartner (Osteuropa, Asien, die islamische Staatenwelt) auszubauen gilt.

2. Der Dialog darf keine Einbahnstraße bleiben. Europa und Nordamerika sind nicht etwa die alleinigen Exporteure des verfassungsstaatlichen Erfolgsmodells, sie können und müssen vielmehr auch von den neuen, in ihren Textgehalten sehr innovativen Normwerken der Staaten in Latein-/Iberoamerika lernen. Das gilt für alle rechtswissenschaftlichen Disziplinen, nicht zuletzt für das Verfassungsrecht.

3. Die Diskussionen in den unterschiedlichen Arbeitgruppen haben eine Gemeinsamkeit herausgearbeitet. Um die Universalität grundlegender Verbürgungen muss immer neu gerungen werden. Sie mag idealiter - etwa mit Blick auf die Menschenrechte - natürlich vorgegeben sein, bedarf realiter aber der immer neuen Konkretisierung im spezifi- 
schen kulturellen Kontexten. Universell ist vor allem die Bedürfnisnatur des Menschen, die in den unterschiedlichen Rechtssystemen bedürfnisadäquate Regelungen verlangt.

4. Nur ein Grundlagendiskurs kann helfen, neue Problemstellungen richtig einzuordnen, gesellschaftlichen Wandel einzufangen. Die tagesaktuellen Fragen sind nur auf der Folie der Grundlagendisziplinen (Rechtsphilosophie, Rechtstheorie, Rechtsgeschichte, Rechtssoziologie, Rechtsanthropologie, Rechtsvergleichung) zu beantworten. Das muss für alle Bereiche rechtswissenschaftlichen Forschens bis hin zu den Curricula der juristischen Fakultäten eindeutige Konsequenzen haben. Jurisprudenz ist immer auch Grundlagenforschung.

5. Zentrale Forschungsfelder dieser Grundlagenarbeit sind heute sowohl in Europa, in den USA, in Latein- und Iberoamerika: Freiheit und öffentliche Sicherheit, Pluralismus in Staat und Gesellschaft, Souveränitätswandel dank staatenübergreifender Integrationsphänomene, die Reform des Sozialstaats, die Harmonisierung divergierender Rechtssysteme und übergreifend eine Gerechtigkeitstheorie, die den Herausforderungen des 21. Jahrhunderts gewachsen ist.

6. Für die Gerechtigkeitsfrage spielt in Latein- und Iberoamerika der Umgang mit den indigenen Rechtskulturen eine besondere Rolle. Hier lassen sich erste gemeinsame Standards für weltweiten Minderheitenschutz, sei es durch verfassungsrechtliche, sei es durch völkerrechtliche Garantien erarbeiten. ${ }^{27}$

7. Latein/Iberoamerika übernimmt schon aufgrund seiner Geschichte eine wichtige Brückenfunktion zwischen dem europäischen und dem amerikanischen Kontinent. In seinen Rechtskulturen verschmelzen wesentlich Elemente von Common Law und Civil Law $^{28}$. Insoweit können die latein-/iberoamerikanischen Rechtstraditionen wichtigen Anstoß geben, wie im Rahmen der europäischen Integration Common Law und kontinentaleuropäisches Rechtsdenken fruchtbar zusammenfinden können. Europa findet sich in der Rolle des Lernenden wieder.

8. Eines vor allem sei abschließend bemerkt: Gerade in der Phase, da Europa um eine Verfassung ringt und sein Verhältnis zu den Vereinigten Staaten neu auszurichten bemüht ist, hilft die latein-/iberoamerikanische Außenperspektive, die oftmals selbstreflexible Bipolarität zwischen Europa und den Vereinigten Staaten, zwischen

J. D. Castrillón (Kolumbien), La organización de Naciones Unidas y los pueblos indígenas : La dinámica del replanteamiento de la visión de las sociedades en el sistema internacional de los derechos humanos. 
Neuer und alter Welt, gewiss auch zwischen „,neuem“ und ,altem“ Europa aufzubrechen. $^{29}$

\section{Ausblick: Die Stimme Iberoamerikas in einem weltumspannenden Dialog der Rechtskulturen}

Der Kongress hat in eindrucksvoller Weise bestätigt, dass die Stimme Iberoamerikas im weltumspannenden „Dialog der Rechtskulturen“ ${ }^{30}$ nachhaltiges Gehör verdient. Ein nach innen gewandter Eurozentrismus auf der einen, ein neuer Unilateralismus der einzig verbleibenden Weltmacht USA auf der anderen Seite führen zu einer gefährlichen Bipolarität. Gerade wo es um den Ausbau einer tragfähigen internationalen Sicherheitsarchitektur geht, die der Präponderanz der Freiheit dennoch Rechnung trägt, wo auf sozialem Sektor die Reformnotwendigkeiten immer drängender werden, wäre ein Denken in Ausschließlichkeiten wenig fruchtbar. Es gilt nicht, das alte Europa gegen die neue Welt in Stellung zu bringen, den Sozialstaat gegen Globalisierungsnotwendigkeiten auszuspielen oder Menschenrechtsuniversalismus gegen die kulturelle Partikularität der Menschenrechte zu stellen. Es geht vielmehr um den schon mehrfach angesprochen Dialog bzw. Diskurs, gerade auch um das Rechtsgespräch zwischen civil law und common law ${ }^{31}$. D. Valadés und dem Instituto de Investigaciones Jurídicas sei dazu auch in Zukunft großer Erfolg und ein neugierig-offenes Ohr all seiner Dialogpartner gewünscht.

Die vielfach kritisierte Trennung von „,altem“ und „neuem“ Europa geht zurück auf eine Formulierung von US-Verteidigungsminister D. Rumsfeld vor einem internationalen Pressecorps am 23. Januar 2003.

So der Titel eines zu Ehren von Peter Häberle am 12. Juli 2002 in Bayreuth veranstalteten internationalen Kolloquiums mit Beiträgen u. a. von $D$. Valadés, The rule of law as a cultural problem, JöR 52 (2004), S. 59 ff.; D. G. Belaunde, Is there a Latin American public space, JöR 52 (2004), S. 59 ff.; $C$. Landa, The Constitutional Reform in Peru as a stage of Constitutional State, JöR 52 (2004), S. 87 ff.; gewichtige ibero- bzw. lateinamerikanische Stimmen in diesem Rechtsdialog sind gewiss auch H. Fix-Zamudio / S. Valencia Carmona, Derecho Constitucional Mexicano y Comparado, Editorial Porrua-UNAM, México 1999; J. Flores / Y. Meyenberg, Ciudadanos y cultura de la democracia, México 2000; M. Alcántara / I. Crespo (eds.), Los límites de la consolidación democrática en America Latina, Salamanca 1995.

Interessant sind hier insbesondere rechtsvergleichend arbeitende Gerichtsentscheidungen, die beide Rechtstraditionen einzubinden suchen, vgl. etwa Valle v. American International Insurance Co., Supreme Court of Puerto Rico, 1979, 108 D. P. R. 692 (Spanisch), 8 P. R. S. Ct. Off. Trans. 735 (English); dazu L. Fiola-Matta, Civil Law and Common Law in the Legal Method of Puerto Rico: Anomalies and Contradictions in Legal Discourse, 24 Cap. U. L. Rev. 153 (1995); B. Schlesinger et al. (eds.), Comparative Law, $6^{\text {th }}$ ed., 1998, p. 192 ff.; für die divergierenden "Common Law Traditions" vgl. schließlich P. de Cruz, Comparative Law in a Changing World, $2^{\text {nd }}$ ed., 1999. 